\title{
Controlling amphiphilic polymer folding beyond the primary structure with protein-mimetic diphenylalanine
}

\author{
Jacqueline L. Warren, Peter A. Dykeman-Bermingham, and Abigail S. Knight* \\ Department of Chemistry, The University of North Carolina at Chapel Hill, Chapel Hill, North Carolina 27599, \\ United States
}

\begin{abstract}
While methods for polymer synthesis have proliferated, their functionality pales in comparison to natural biopolymers - strategies are limited for building the intricate network of noncovalent interactions necessary to elicit complex, protein-like functions. Using a bioinspired diphenylalanine acrylamide (FF) monomer, we explored the impact of various non-covalent interactions in generating ordered assembled structures. Amphiphilic copolymers were synthesized that exhibit $\beta$-sheet-like secondary structure upon collapsing into single-chain assemblies in aqueous environments. Systematic analysis of a series of amphiphilic copolymers illustrated that the collapse is primarily driven by hydrophobic forces. Hydrogen-bonding and aromatic interactions stabilize local structure, as $\beta$-sheet-like interactions were identified via circular dichroism and thioflavin $\mathrm{T}$ fluorescence. Similar analysis of phenylalanine $(\mathrm{F})$ and alanine-phenylalanine acrylamide (AF) copolymers found that distancing the aromatic residue from the polymer backbone is sufficient to induce $\beta$-sheet-like secondary structure akin to the FF copolymers; however, the interactions between AF subunits are less stable than those formed by FF. Further, hydrogen-bond donating hydrophilic monomers disrupt internal structure formed by FF within collapsed assemblies. Collectively, these results illuminate design principles for the facile incorporation of multiple facets of protein-mimetic, higher-order structure within folded synthetic polymers.
\end{abstract}

\section{INTRODUCTION}

Confined to a limited pool of natural amino acid monomers, proteins have evolved primary structures that yield canonical structure hierarchy: quaternary structures of multiple polypeptide chains, tertiary structure that defines the three-dimensional morphology, and local rigid regions dictated by secondary structure. Polymer chemists have far outpaced natural proteins in increasing the diversity of monomers; yet, establishing the complex functions of natural biomacromolecules, and thus the intricate networks of structure-driving non-covalent interactions is a remaining challenge necessary to expand the functionality of next generation materials. ${ }^{1-3}$ Each hierarchical level of folding is required to create subdomains capable of protein-mimetic functions such as specificity, catalysis, and allostery.

Despite this need for higher-order structure, there are few examples of synthetic polymer assemblies that demonstrate these motifs in an aqueous environment. ${ }^{4}$ Protein-mimetic collapsed tertiary structure has been generated with hydrogen-bonding using a pendent benzene-1,3,5-tricarboxamide (BTA) moiety, ${ }^{5-8}$ hydrophobic collapse using alkyl chains, ${ }^{9-14}$ and covalent crosslinks ${ }^{15}$. As secondary structure is driven by backbone hydrogen bonding in proteins, it is challenging to mimic with an amide-lacking polymer backbone. Helices can be introduced with the pendent BTA moiety methacrylate monomer that is incorporated into a random amphiphilic copolymer through ruthenium-catalyzed living radical polymerization. ${ }^{5,6}$ However, the synthetic versatility of the polymerizable BTA methacrylate is not currently available for $\beta$-sheets, which have only been introduced into aqueous assemblies through incorporation of a peptide block into the polymer backbone. Oligoleucine has been used as an initiator in nitroxide-mediated radical polymerization placing a peptide in the backbone of a polymer and 
leading to the formation of $\beta$-sheet regions. ${ }^{16,17}$ These strategies provide a foundation for the introduction of local structure into polymer scaffolds, but we lack a full range of tools to create secondary structure in synthetic polymers - i.e., moieties that can be easily utilized alongside the diverse scope of monomers already in the polymer chemist's toolbox.

Diphenylalanine, a moiety identified to drive secondary structure formation in amyloid fibrils, has been implemented across disciplines (e.g., drug delivery, hydrogel formation, and metal-organic frameworks) to lead to aggregation in aqueous environments including driving multi-chain assembly of hydrophilic PEG or peptides. ${ }^{18-21}$ Isolated di- and tripeptides containing diphenylalanine have shown that hydrogen bonding and aromatic interactions stabilize aggregated structures as characterized with circular dichroism (CD) and Fourier transform infrared spectroscopy. ${ }^{22,23}$ Motivated by these observations, we hypothesized that diphenylalanine could generate higher-order structure in an amphiphilic copolymer. Herein we demonstrate the role of aromatic interactions and hydrogen bonding in the formation of tertiary structure and novel $\beta$-sheet-like secondary structure induced through a pendent moiety.

\section{RESULTS AND DISCUSSION}

Diphenylalanine Monomer (FF) Synthesis and Copolymerization. Motivated by the unique propensity of diphenylalanine to drive $\beta$-sheet formation, we synthesized a bioinspired monomer with a pendent diphenylalanine moiety (Figure 1a). A diphenylalanine acrylamide monomer was designed (methyl acryloyl di-L-phenylalanine, FF; Figure 1b) to be compatible with common controlled radical polymerizations, which provide monomer scope versatility, chain-end
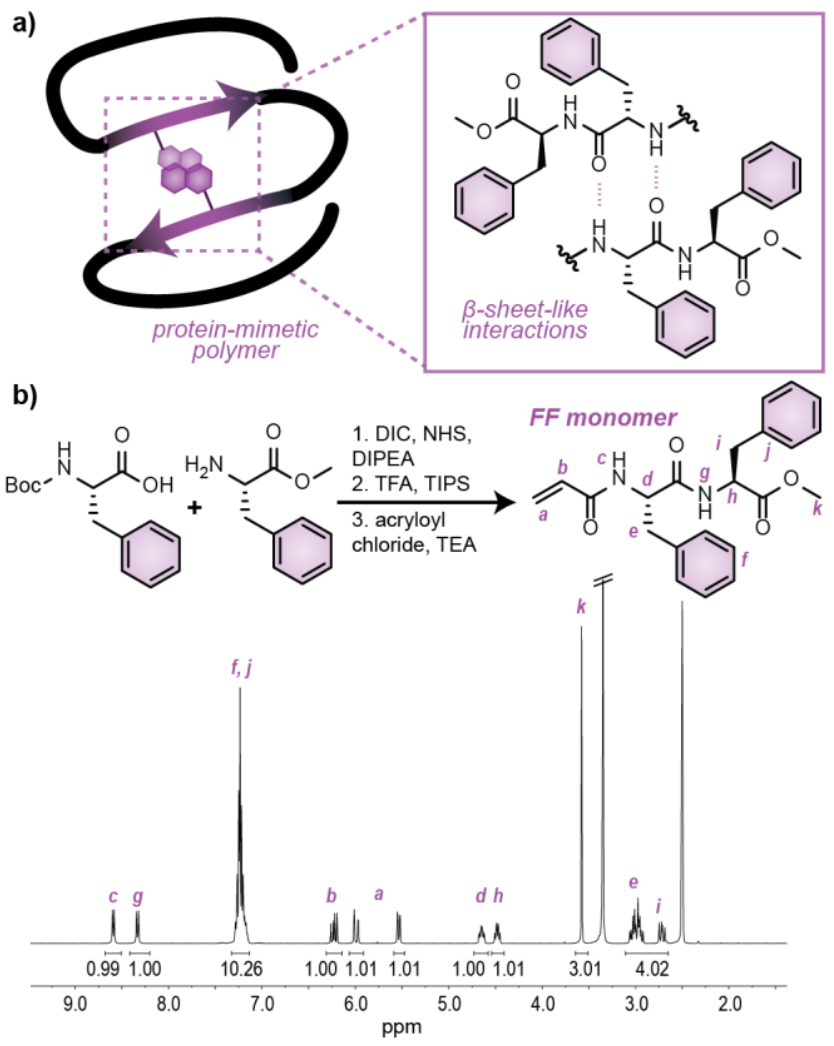

Figure 1. Diphenylalanine acrylamide monomer. a) Schematic illustrating the hydrogen-bonding and aromatic interactions leading to $\beta$-sheet-like secondary structure in amphiphilic copolymers. b) Schematic of the FF monomer 3-step synthesis and resulting ${ }^{1} \mathrm{H}$ NMR of the purified product in DMSO- $d_{6}$. 
fidelity, and control of the polymer molecular weight and dispersity. ${ }^{24,25}$ The synthesis was accomplished using an amide coupling of two orthogonally protected L-phenylalanines ( $N$-Boc and methyl ester) followed by deprotection of the Boc and substitution of acryloyl chloride (characterization in Figures S1-6).

Inspired by protein-mimetic statistical copolymers with helical domains ${ }^{5}$ and the natural separation of $\beta$-sheet forming residues in protein sequences, we synthesized random amphiphilic copolymers with diphenylalanine moieties distributed throughout the polymer scaffold. Dimethylacrylamide (DMA) was selected as a hydrophilic monomer as it lacks a hydrogen bond donor and thus is unlikely to disrupt hydrogen-bonding between diphenylalanine moieties. Due to the steric bulk of FF and conformational restraints imposed by its multiple amide bonds, we assessed the ability of the FF monomer to achieve an approximately statistical distribution within the copolymer backbone and a Gaussian molecular weight distribution when copolymerized with DMA. Reversible addition-fragmentation chain transfer (RAFT) polymerization ${ }^{26}$ performed in DMF yielded polymers with controlled molecular weight $(M n)$, low dispersity $(\bigoplus)$, and similar rates of monomer incorporation (Figures S7-10 and Tables S1 and S2).

Tertiary Structure: Characterization of Polymer Collapse and Assembly. To investigate the assembled conformation of FF-DMA copolymers, a series ( 0 to $40 \mathrm{wt} \% \mathrm{FF}$ ) were synthesized and their morphologies in neutral aqueous solution (100 mM phosphate, $\mathrm{pH}$ 7) were characterized. To probe the collapse in an aqueous environment, three methods of assembly were employed: dissolution, sonication, and a heat-cool cycle before characterization with dynamic light scattering (DLS) (Figure 2a and Table S3). For copolymers containing $20 \mathrm{wt} \%$ or less of FF, single-chain nanoparticles (SCNPs) of comparable sizes were observed for all assemblies. Aggregation was visible for 30 and 40 wt $\%$ FF in the dissolved copolymers; however, both sonication and a heat-cool cycle led to the formation of SCNPs. DLS enabled the rapid identification of single chains and suggested a limited impact of the assembly strategy on the aggregation state. However, it was challenging to quantify the anticipated increase in collapse with increasing hydrophobicity due to the limited instrument accuracy at low hydrodynamic radii.

To calculate percent collapse that correlated with the hydrophobic weight percent of the copolymers, we compared the compact and swollen states of the amphiphilic polymers using size exclusion chromatography (SEC) in aqueous and organic (DMF) solvents (Figures $2 \mathrm{~b}$ and S11), a strategy used by Sawamoto and coworkers. ${ }^{27}$ By calibrating with poly(ethylene)glycol standards in each solvent, a ratio of the peak apex molecular weight $\left(M_{P}\right)$ in aqueous buffer $\left[M_{P(a q)}\right]$ and DMF $\left[M_{P(D M F)}\right]$ is a measure of compactness (Figures S12 and S13). We converted this value to a percent by multiplying by 100 and plotted this percent compaction as a function of the FF wt\% (Figure 2). Using this strategy, we were able to observe the anticipated linear relationship and over 50\% collapse at $40 \mathrm{wt} \%$ FF (Table S4). Although the more hydrophobic polymers formed multi-chain aggregates as characterized by DLS, the polymers were diluted during SEC characterization yielding single chains as characterized by multi-angle light scattering (MALS; Figure S14). Additionally, three separate 20 wt\% FF polymers were synthesized and yielded the same percent collapse values for similar molecular weights demonstrating reproducible collapse characteristics (Figure S15). Percent collapse was observed to decrease as the molecular weight was altered over a range of 7-54 kDa for FF copolymers. (Figure 16, Table S5).

To probe the factors contributing to the collapse of protein-mimetic FF-DMA, we designed an analogous series of amphiphilic DMA copolymers with benzyl acrylamide (BAA) and methyl L-phenylalanine (F) with lower propensity to hydrogen bond and non-aromatic tert-butyl acrylamide (tBA) (Figures 2c and S17-S25 and Tables S6 and S7). Both hydrophobicity and hydrogen bonding are known to contribute to protein stability, ${ }^{28,29}$ and diphenylalanine is known to have 
strong hydrogen-bonding and hydrophobic interactions. ${ }^{18,30}$ Thus either interaction or a combination could be responsible for the observed collapse.

The three aromatic monomers led to similar collapse percentages, leading to the hypothesis that the collapse is primarily driven by hydrophobicity (Figures $2 \mathrm{~d}$ and $\mathrm{S} 11, \mathrm{~S} 26-28$ ). To further investigate this, the ratio of collapse to $\log \mathrm{P}^{31,32}$ (estimated by Marvin Sketch) was plotted as a function of the mole percentage of the hydrophobic monomer (Figure S29). Linear regressions revealed similar relationships for FF-DMA, F-DMA, and BAA-DMA and showed lower collapse percentages than the $\log \mathrm{P}$ would predict for tBA-DMA (Table S8). We hypothesize this difference is due to the bulkiness of tBA close to the backbone, and that the collapse is predominantly driven by hydrophobicity.

a)

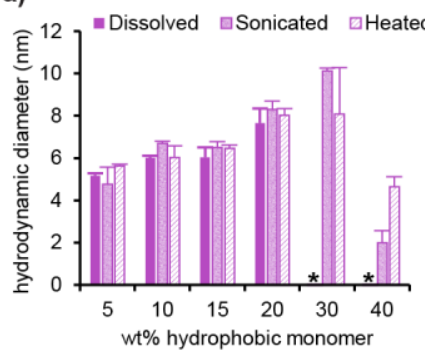

b)

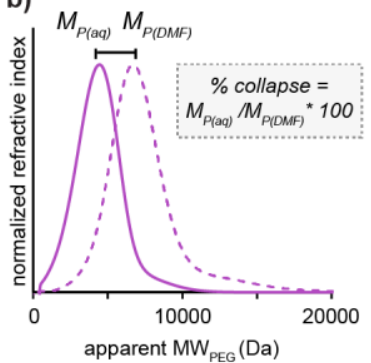

c)

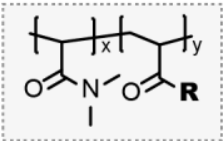

$\mathbf{R}=$

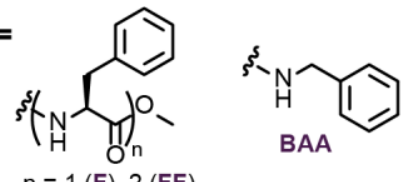

$\mathrm{n}=1(\mathrm{~F}), 2$ (FF)

d)

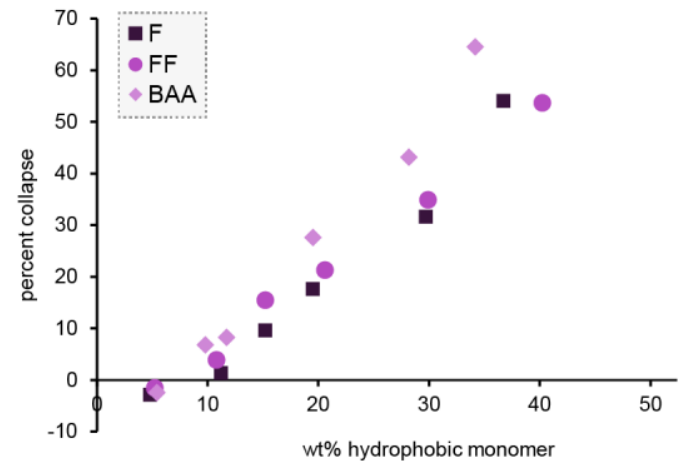

Figure 2. Assembled structure of FF-DMA copolymers. a) DLS of FF-DMA copolymers at various wt $\%$ s when dissolved, sonicated for $1 \mathrm{~h}$, or heated for $30 \mathrm{~min}$ and slowly cooled to r.t. in phosphate buffer $(100 \mathrm{mM}, \mathrm{pH} 7)$ at a polymer concentration of $10 \mathrm{mg} / \mathrm{mL}$. Asterisks (*) represent multichain aggregation where the hydrodynamic diameter was measured as $>100 \mathrm{~nm}$. Error bars represent standard deviations $(n=3)$. b) Calculation of percent collapse by converting retention time (min) into apparent molecular weight of PEG (MW $\left.\mathrm{MEG}_{\mathrm{PE}}\right)$ using a PEG standard curve run in both solvent systems (aqueous and DMF). The peak apex molecular weight $\left(M_{P}\right)$ was determined and a ratio was calculated. c) Amphiphilic polymers compared to FF-DMA. d) Percent collapse plotted as a function of $\mathrm{wt} \%$.

Secondary Structure: Characterization of Local Polymer Structure. While collapse is a key component of proteinmimetic polymer structure, local secondary structure is also critical for complex functions; this is what motivated the 
selection of the diphenylalanine moiety. Circular dichroism (CD) spectroscopy and differential scanning calorimetry (DSC) both revealed more local order in FF-containing copolymers compared to F copolymers. As anticipated, polymers composed solely of achiral monomers had no observable CD spectra (Figures 3a and S30). Unique CD spectra were observed for DMA copolymers with FF and $\mathrm{F}$ in aqueous solution (100 mM phosphate, $\mathrm{pH} 7)$. Although $\beta$-sheets commonly display minima, the high percentage of aromatic amino acids generates non-canonical spectra. The maximum at $198 \mathrm{~nm}$ is indicative of $\pi-$ $\pi^{*}$ transitions caused by aromatic stacking, while the peak at $217 \mathrm{~nm}$ is described as an $\mathrm{n}-\pi^{*}$ transition originating from hydrogen bonding. ${ }^{22}$ The intensities of the peaks at $217 \mathrm{~nm}$ for both F- and FF-DMA copolymers are similar, suggesting similar quantities of hydrogen bonds in chiral environments in both collapsed structures when controlling for molecular weight (Figure 3a and S31). However, the FF copolymer has both a narrower peak than the F copolymer at $217 \mathrm{~nm}$ and a higher intensity peak at $198 \mathrm{~nm}$, both indicating a more ordered structure. ${ }^{33}$ As the molecular weight was altered over a range from 7-54 kDa, the spectra remained comparable for $20 \mathrm{wt} \%$ FF copolymers with DMA (Figure S32). Heating the aqueous polymer assemblies to $90{ }^{\circ} \mathrm{C}$ decreases both maxima, as is commonly observed for intramolecular interactions (Figures $3 \mathrm{~b}$ and S33). ${ }^{34}$ Characterization of the copolymers with DSC additionally demonstrates stronger intramolecular interactions, and thus more ordered local structures for FF-DMA: the FF-DMA copolymer with $30 \mathrm{wt} \%$ FF has a higher glass transition temperature $\left(\mathrm{T}_{\mathrm{g}}\right)$ than the DMA homopolymer. Conversely, the F-DMA (30 wt \% F) copolymer has a lower $\mathrm{T}_{\mathrm{g}}$ than the DMA homopolymer (Table S9).

Further investigation of polymer internal structure with thioflavin T (ThT) fluorescence spectroscopy indicates $\beta$-sheet-like interactions (Figure 3c). Emission at $480 \mathrm{~nm}$ is commonly observed for amyloid aggregates and $\beta$-sheet-like interactions..$^{21,35,36}$ Copolymers of increasing weight percentages of $\mathrm{FF}$ and $\mathrm{F}$ demonstrated increasing fluorescence with increasing percent hydrophobicity. The $30 \mathrm{wt} \%$ samples highlight that FF-DMA is forming more ordered $\beta$-sheet-like structures in single-chain assemblies.
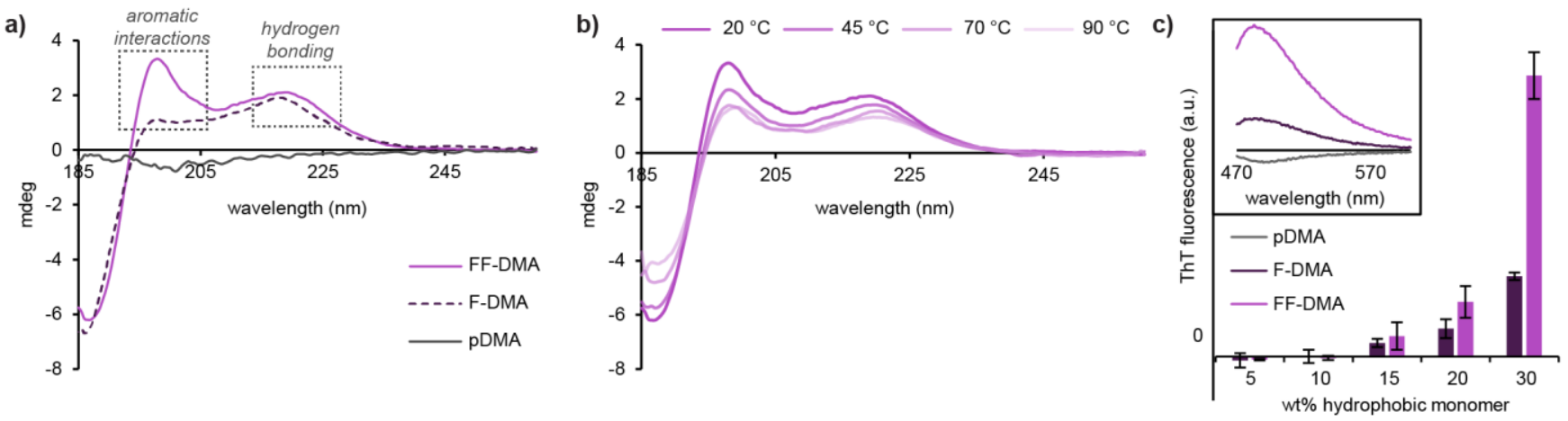

Figure 3. Secondary structure of FF-DMA copolymers. a) CD spectra of $20 \mathrm{wt} \%$ F- and FF-DMA polymers compared to a DMA homopolymer (pDMA) at $20^{\circ} \mathrm{C}$. b) Temperature dependence of CD spectra for $20 \mathrm{wt} \%$ FF-DMA polymer taken at $20-90{ }^{\circ} \mathrm{C}$. c) ThT fluorescence of F- and FF-DMA at varying wt $\%$ s. Inset shows the fluorescence spectra of $30 \mathrm{wt} \% \mathrm{~F}$ - and FF-DMA polymers compared to pDMA. Error bars represent standard deviation $(n=3-5)$. All characterization was performed in $1 \mathrm{mM}$ phosphate buffer at $\mathrm{pH} 7$.

Positioning F Farther from the Backbone: Alanine-Phenylalanine Monomer (AF). While the fibrilization of diphenylalanine-containing molecules has been thoroughly characterized, phenylalanine alone and other phenylalaninecontaining dipeptides have also demonstrated some of the hallmark characteristics of assembly including fibrilization and similar CD spectra similar to diphenylalanine. ${ }^{18,33,37}$ To investigate whether the diphenylalanine moiety or simply the presence of a phenylalanine distanced from the backbone led to the increased internal structure, an alanine-phenylalanine acrylamide was synthesized and characterized (methyl acryloyl L-alanyl-L-phenylalanine, AF; Figures 4a and S34-S40). 
Collapse of AF-DMA copolymers was measured as described for FF-DMA previously, and trends similar to those of the previous copolymers with aromatic sidechains were observed (Figure S41 and Table S10). When plotted as a function of mole percent of the hydrophobic monomer, the ratio of the $\log \mathrm{P}$ value to the percent collapse was higher than that observed for FF and F (Figure S42 and Table S11), suggesting the flexibility of the alanine linker led to more collapsed structures than predicted by the hydrophobicity.

The internal structure of the collapsed AF copolymers resembles that of the FF copolymers, indicating the AF copolymers also contain $\beta$-sheet-like regions. Fluorescence in the presence of ThT was compared among polymers with similar compactness (30 wt\% hydrophobic monomer, 1 and 2 in Figure 4a,b) and among those with comparable total numbers of phenylalanine residues (15 mol\% F, 1 and 3 in Figure 4a,b). Copolymers with equivalent weight percents have similar numbers of intramolecular physical crosslinks, and the FF-DMA copolymers display more ThT fluorescence. The polymers with equivalent mole percents have similar numbers of total phenylalanine residues, and the AF-DMA polymers display a higher ThT fluorescence likely due to the increased number of intramolecular physical crosslinks as the polymer is two times more compact. The presence of ordered local structure is supported by the CD spectrum that mirrors that of the FF-DMA copolymers (Figure 4c - both 20 wt\% hydrophobic monomer and Figure S43). However, heating both samples to $90{ }^{\circ} \mathrm{C}$ revealed the AF driven collapse was less stable as the ellipticity decreases more than that of the FF-DMA copolymers. This is supported by the minimal difference in $\mathrm{T}_{\mathrm{g}}$ observed for AF-DMA copolymers as compared to pDMA, as opposed to the increase observed for FF-DMA (Table S12).

a)
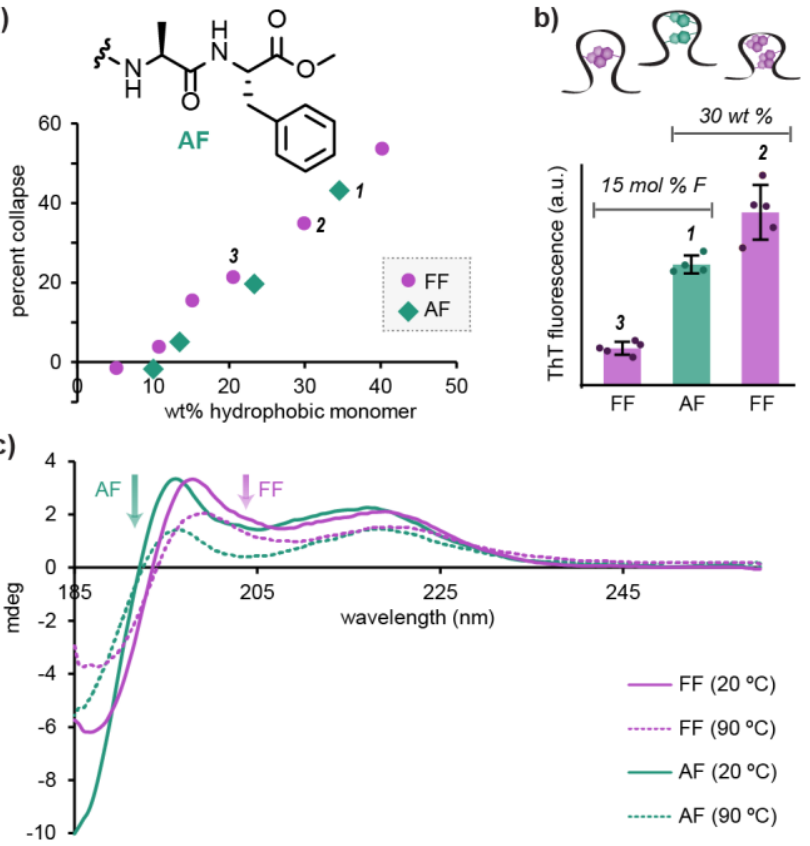

.


Exploring the Impact of Hydrogen-Bond Donors within Hydrophilic Monomers. After probing the impact of systematic variation of the hydrophobic monomer, we sought to investigate how the presence of hydrogen bond donors in the hydrophilic monomer would influence the folded polymer structure. FF-hydrophilic acrylamide amphiphilic copolymers (20 wt\% FF) were synthesized with $N$-hydroxyethyl acrylamide (HEAA) and acrylamide (AM) (Figures 5a, S44, and S45). The amphiphilic copolymers of comparable molecular weights were characterized via aqueous SEC alone as acrylamide homopolymers are insoluble in DMF.

As anticipated, due to the difference in hydrophilicity and additional intramolecular hydrogen bonds, HEAA and AM containing copolymers were more collapsed than the original F- and FF-DMA copolymers (Figures 5b and S46-49; Table S13). Fluorescence of ThT is also enhanced in the more collapsed HEAA and AM copolymers, which suggests the additional hydrogen-bond donors stabilize the $\beta$-sheet-like interactions (Figure 5c and S50).

CD spectra provide additional insight into the intramolecular interactions. Near $218 \mathrm{~nm}$, the narrowest peak is FF-DMA as compared to FF-HEAA and -AM, suggesting the DMA copolymer, which lacks additional hydrogen bond donors, has the most rigid internal structure (Figure 5d). Copolymers with HEAA and AM no longer have a peak in the $198 \mathrm{~nm}$ region. Amide- $\pi$ interactions have been frequently observed in proteins and can form stronger interactions than amide hydrogen bonds; ${ }^{38,39}$ thus, amide- $\pi$ interactions may be disrupting the aromatic interactions observed in FF-DMA. This suggests that while HEAA and AM copolymers lead to more collapsed structures, there are fewer interactions between the diphenylalanine moieties in FF-HEAA and -AM copolymers.

a)

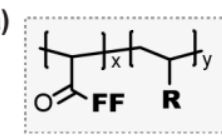

b)

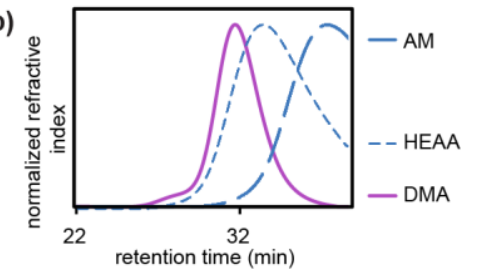

$\mathbf{R}=$

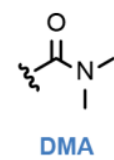

MA d)

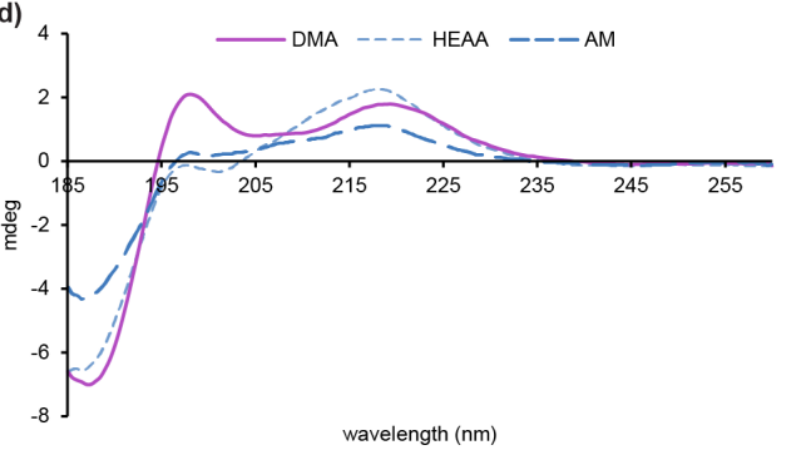

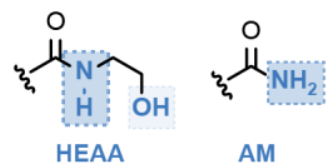

c)

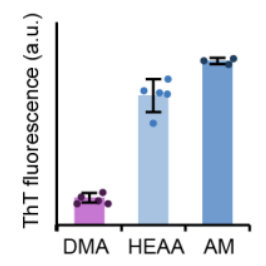

Figure 5. Evaluating the impact of hydrogen-bond donors within hydrophilic monomers. a) Copolymer structure of FF-DMA, HEAA, and -AM. b) Comparison of SEC of $20 \mathrm{wt} \%$ FF-DMA, HEAA, and -AM. Comparison of c) ThT fluorescence and d) CD spectra of $20 \mathrm{wt} \%$ FF-DMA, -HEAA, and -AM. Error bars represent standard deviation $(n=3-5)$. 


\section{CONCLUSIONS}

We have described a series of amphiphilic copolymers with tunable tertiary and secondary structure. By comparing the hydrophobic collapse of amino acid based hydrophobic monomers (FF, AF, and F) and commercial monomers (BAA and tBA) we determined that the amount of collapse in copolymers with DMA is predominantly dependent on the weight percent of the hydrophobic monomer. However, internal structure, as characterized by ThT fluorescence and CD, depends on the composition of the hydrophobic monomer and the presence or absence of hydrogen-bond donors in the hydrophilic comonomer. The FF-DMA copolymer generates the most ordered intramolecular interactions, while FF-AM leads to the highest collapse with additional hydrogen bond donors.

This suite of amphiphilic copolymers opens the door for the development of synthetic single-chain aqueous assemblies with both helical and $\beta$-sheet-like domains, continuing to close the gap between synthetic polymers and biomacromolecules. Additionally, studies with non-random copolymers could reveal control over tertiary and quaternary structure including controlling the number of chains in multi-chain assemblies and increasing the number of $\beta$-sheet-like interactions. This tunability will facilitate the development of biocompatible nanomaterials with complex functions such as selective binding, catalysis, or allostery that rival and improve upon the capabilities of natural proteins.

\section{AUTHOR INFORMATION}

Corresponding Author

* Abigail S. Knight (aknight@unc.edu)

\section{ACKNOWLEDGMENT}

This material is based upon work supported by the U.S. Department of Energy, Office of Science, Office of Basic Energy Sciences under Award Number DE-SC0021295. J.L.W. thanks UNC Department of Chemistry's E.C Markham Summer Research Award for a summer undergraduate fellowship. We acknowledge the Macromolecular Interactions Facility (SECMALS and CD; supported by the National Cancer Institute of the National Institutes of Health under award number P30CA016086), the UNC Department of Chemistry Mass Spectrometry Core Laboratory (HRMS; supported by the National Cancer Institute of the National Institutes of Health under award number P30CA016086 and the National Science Foundation under grant numbers CHE-0922858 and CHE-1726291), and the Redinbo (ClarioStar plate reader), Leibfarth (GPC, TGA, and DSC), and Dick (DLS) laboratories for assistance with instrumentation. Additionally, the NMR Core Laboratory staff, Drs. Marc ter Horst and Andrew Camp, for feedback on NMR characterization.

\section{REFERENCES}

(1) Frisch, H.; Tuten, B. T.; Barner-Kowollik, C. Macromolecular Superstructures: A Future Beyond Single Chain Nanoparticles. Isr. J. Chem. 2020, 60 (1-2), 86-99.

(2) Liu, L.; Gellman, S. H. Harnessing Noncovalent Interactions to Drive Single-Chain Nanoparticle Formation. Macromolecules 2020, 53 (19), 8141-8143.

(3) Hanlon, A. M.; Lyon, C. K.; Berda, E. B. What Is Next in Single-Chain Nanoparticles? Macromolecules 2016, 49 (1), 2-14.

(4) Barbee, M. H.; Wright, Z. M.; Allen, B. P.; Taylor, H. F.; Patteson, E. F.; Knight, A. S. Protein-Mimetic Self-Assembly with Synthetic Macromolecules. Macromolecules 2021, acs.macromol.0c02826.

(5) Terashima, T.; Mes, T.; De Greef, T. F. A.; Gillissen, M. A. J.; Besenius, P.; Palmans, A. R. A.; Meijer, E. W. Single-Chain Folding of Polymers for Catalytic Systems in Water. J. Am. Chem. Soc. 2011, 133 (13), 4742-4745. 
Chains. Macromolecules 2013, 46 (10), 4120-4125.

Huerta, E.; Stals, P. J. M.; Meijer, E. W.; Palmans, A. R. A. Consequences of Folding a Water-Soluble Polymer Around an Organocatalyst. Angew. Chemie 2013, 125 (10), 2978-2982.

Artar, M.; Terashima, T.; Sawamoto, M.; Meijer, E. W.; Palmans, A. R. A. Understanding the Catalytic Activity of Single-Chain Polymeric Nanoparticles in Water. J. Polym. Sci. Part A Polym. Chem. 2014, 52 (1), 12-20.

Hirai, Y.; Terashima, T.; Takenaka, M.; Sawamoto, M. Precision Self-Assembly of Amphiphilic Random Copolymers into Uniform and Self-Sorting Nanocompartments in Water. Macromolecules 2016, 49 (14), 5084-5091.

(10) Kimura, Y.; Imai, S.; Takenaka, M.; Terashima, T. Amphiphilic Random Cyclocopolymers as Versatile Scaffolds for RingFunctionalized and Self-Assembled Materials. Macromolecules 2021, 54 (9), 3987-3998.

(11) Kimura, Y.; Ouchi, M.; Terashima, T. Folded Amphiphilic Homopolymer Micelles in Water: Uniform Self-Assembly beyond Amphiphilic Random Copolymers. Polym. Chem. 2020, 11 (32), 5156-5162.

(12) Hattori, G.; Takenaka, M.; Sawamoto, M.; Terashima, T. Nanostructured Materials via the Pendant Self-Assembly of Amphiphilic Crystalline Random Copolymers. J. Am. Chem. Soc. 2018, 140 (27), 8376-8379.

(13) Imai, S.; Hirai, Y.; Nagao, C.; Sawamoto, M.; Terashima, T. Programmed Self-Assembly Systems of Amphiphilic Random Copolymers into Size-Controlled and Thermoresponsive Micelles in Water. Macromolecules 2018, 51 (2), 398-409.

Shibata, M.; Matsumoto, M.; Hirai, Y.; Takenaka, M.; Sawamoto, M.; Terashima, T. Intramolecular Folding or Intermolecular SelfAssembly of Amphiphilic Random Copolymers: On-Demand Control by Pendant Design. Macromolecules 2018, 51 (10), 3738-3745. Chen, J.; Wang, J.; Li, K.; Wang, Y.; Gruebele, M.; Ferguson, A. L.; Zimmerman, S. C. Polymeric "Clickase" Accelerates the Copper Click Reaction of Small Molecules, Proteins, and Cells. J. Am. Chem. Soc 2019, 141, 9700.

Nishimura, S. N.; Higashi, N.; Koga, T. Synthesis of Peptide-Vinyl Polymer Multiblock Hybrids by Nitroxide-Mediated Polymerization: Breaking the Limitations of Monomer Compatibility. Polym. Chem. 2019, 10 (1), 71-76.

Nishimura, S.; Higashi, N.; Koga, T. A Novel Thermo-Responsive Multiblock Architecture Composed of a Sequential Peptide and an Amino Acid-Derived Vinyl Polymer: Toward Protein-Mimicking Single-Chain Folding. Chem. Commun. 2019, 55 (10), $1498-1501$. Adler-Abramovich, L.; Gazit, E. The Physical Properties of Supramolecular Peptide Assemblies: From Building Block Association to Technological Applications. Chem. Soc. Rev. 2014, 43 (20), 6881-6893.

Diaferia, C.; Roviello, V.; Morelli, G.; Accardo, A. Self-Assembly of PEGylated Diphenylalanines into Photoluminescent Fibrillary Aggregates. ChemPhysChem 2019, 20 (21), 2774-2782.

Guo, C.; Luo, Y.; Zhou, R.; Wei, G. Probing the Self-Assembly Mechanism of Diphenylalanine-Based Peptide Nanovesicles and Nanotubes. ACS Nano 2012, 6 (5), 3907-3918.

Castelletto, V.; Hamley, I. W. Self Assembly of a Model Amphiphilic Phenylalanine Peptide/Polyethylene Glycol Block Copolymer in Aqueous Solution. Biophys. Chem. 2009, 141 (2-3), 169-174.

Mishra, A.; Chauhan, V. S. Probing the Role of Aromaticity in the Design of Dipeptide Based Nanostructures. Nanoscale 2011, 3 (3), 945-949.

Bera, S.; Mondal, S.; Xue, B.; Shimon, L. J. W.; Cao, Y.; Gazit, E. Rigid Helical-like Assemblies from a Self-Aggregating Tripeptide. Nat. Mater. 2019, 18 (5), 503-509.

Parkatzidis, K.; Zurich, E.; Anastasaki, A. Recent Developments and Future Challenges in Controlled Radical Polymerization: A 2020 Update. 2020.

Grubbs, R. B.; Grubbs, R. H. 50th Anniversary Perspective: Living Polymerization - Emphasizing the Molecule in Macromolecules. Macromolecules. American Chemical Society September 26, 2017, pp 6979-6997.

Perrier, S. S. 50th Anniversary Perspective: RAFT Polymerization-A User Guide. Macromolecules 2017, 50, 7433-7447.

Matsumoto, K.; Terashima, T.; Sugita, T.; Takenaka, M.; Sawamoto, M. Amphiphilic Random Copolymers with

Hydrophobic/Hydrogen-Bonding Urea Pendants: Self-Folding Polymers in Aqueous and Organic Media. Macromolecules 2016, 49 (20), 7917-7927.

Pace, C. N.; Scholtz, J. M.; Grimsley, G. R. Forces Stabilizing Proteins. FEBS Lett. 2014, 588 (14), 2177-2184.

Pace, C. N.; Fu, H.; Fryar, K. L.; Landua, J.; Trevino, S. R.; Shirley, B. A.; Hendricks, M. M. N.; Iimura, S.; Gajiwala, K.; Scholtz, J. M.; et al. Contribution of Hydrophobic Interactions to Protein Stability. J. Mol. Biol. 2011, 408 (3), 514-528.

(30) Guo, C.; Luo, Y.; Zhou, R.; Wei, G. Probing the Self-Assembly Mechanism of Diphenylalanine-Based Peptide Nanovesicles and 
Nanotubes. ACS Nano 2012, 6 (5), 3907-3918.

(31) Foster, J. C.; Akar, I.; Grocott, M. C.; Pearce, A. K.; Mathers, R. T.; O’Reilly, R. K. 100th Anniversary of Macromolecular Science Viewpoint: The Role of Hydrophobicity in Polymer Phenomena. ACS Macro Lett. 2020, 9 (11), 1700-1707.

(32) Mannhold, R.; Poda, G. I.; Ostermann, C.; Tetko, I. V. Calculation of Molecular Lipophilicity: State-of-the-Art and Comparison of LogP Methods on More than 96,000 Compounds. J. Pharm. Sci. 2009, 98 (3), 861-893.

(33) Amdursky, N.; Stevens, M. M. Circular Dichroism of Amino Acids: Following the Structural Formation of Phenylalanine.

ChemPhysChem 2015, 16 (13), 2768-2774.

(34) Greenfield, N. J. Using Circular Dichroism Collected as a Function of Temperature to Determine the Thermodynamics of Protein Unfolding and Binding Interactions. Nat. Protoc. 2007, 1 (6), 2527-2535.

(35) Biancalana, M.; Koide, S. Molecular Mechanism of Thioflavin-T Binding to Amyloid Fibrils. Biochimica et Biophysica Acta - Proteins and Proteomics. NIH Public Access July 2010, pp 1405-1412.

(36) Krysmann, M. J.; Castelletto, V.; Hamley, I. W. Fibrillisation of Hydrophobically Modified Amyloid Peptide Fragments in an Organic Solvent. Soft Matter 2007, 3 (11), 1401-1406.

(37) Reddy, S. M. M.; Shanmugam, G. Role of Intramolecular Aromatic $\pi-\pi$ Interactions in the Self-Assembly of Di-1-Phenylalanine Dipeptide Driven by Intermolecular Interactions: Effect of Alanine Substitution. ChemPhysChem 2016, 17 (18), $2897-2907$.

(38) Tóth, G.; Murphy, R. F.; Lovas, S. Investigation of Aromatic-Backbone Amide Interactions in the Model Peptide Acetyl-Phe-Gly-GlyN-Methyl Amide Using Molecular Dynamics Simulations and Protein Database Search. J. Am. Chem. Soc. 2001, 123 (47), 1178211790 .

(39) Steiner, T.; Koellner, G. Hydrogen Bonds with $\pi$-Acceptors in Proteins: Frequencies and Role in Stabilizing Local 3D Structures. $J$. Mol. Biol. 2001, 305 (3), 535-557. 